\title{
One Electrogram-Tracing Tells All: what is the mechanism of this supraventricular tachycardia?
}

\author{
Moyuru Hirata ${ }^{1}$, Yuji Wakamatsu ${ }^{1}$, Koichi Nagashima ${ }^{2}$, Sayaka Kurokawa ${ }^{2}$, Naoto \\ Otsuka $^{2}$, Seina Yagyu ${ }^{2}$, Shu Hirata ${ }^{3}$, Toshiko Nakai ${ }^{4}$, and Yasuo Okumura ${ }^{2}$ \\ ${ }^{1}$ Nihon University School of Medicine Graduate School of Medicine \\ ${ }^{2}$ Nihon University School of Medicine \\ ${ }^{3}$ Nihon University School of Medicine, Division of Cardiology, Department of Medicine \\ ${ }^{4}$ Division of Cardiology,
}

January 21, 2021
One Electrogram-Tracing Tells All: what is the mechanism of this supraventricular tachycar- dia?
Moyuru Hirata, MD*, Yuji Wakamatsu, MD*, Koichi Nagashima, MD, PhD*, Sayaka Kurokawa, MD, PhD*, Naoto Otsuka, MD*, Seina Yagyu, MD*, Shu Hirata, MD*, Toshiko Nakai, MD, PhD*, Yasuo Okumura, $\mathrm{MD}, \mathrm{PhD}$.
*Division of Cardiology, Department of Medicine, Nihon University School of Medicine, Tokyo, Japan
Correspondence

Koichi Nagashima, MD, PhD; Division of Cardiology, Department of Medicine, Nihon University School of Medicine, 30-1 Ohyaguchi-kamicho, Itabashi-ku, Tokyo 173-8610, Japan

Tel: +81-3-3972-8111

Fax: +81-3-3972-1098

E-mail: cocakochan@gmail.com

Total word count: 1036 words, 2 Figures

Funding: (None)

Disclosures: (None)

The work described was supported by departmental resources only.

Keywords: narrow QRS tachycardia, atrioventricular nodal reentrant tachycardia, accessary pathway.

\section{Case presentation}

A 72-year-old man without overt structural heart disease was referred to our institution for catheter ablation of a narrow QRS tachycardia. A 12-lead electrocardiogram recorded during sinus rhythm showed no ventricular preexcitation. Multielectrode catheters were placed in the high right atrium, His bundle (HB) region, coronary sinus (CS), and right ventricular (RV) apex. During sinus rhythm, the cycle length (CL), atrio-His interval, and His-ventricular interval were 758, 102, and $52 \mathrm{~ms}$, respectively. A narrow QRS complex tachycardia with a CL of $270 \mathrm{~ms}$ was induced without atrio-His jump by atrial extrastimulation. The 
intracardiac electrograms with RV overdrive pacing during the tachycardia are shown in Figure 1A-B. What is the mechanism of this tachycardia?

\section{Discussion}

The tachycardia exhibited the earliest atrial activation at the HB region with a ventriculo-atrial interval of $-6 \mathrm{~ms}$, i.e., an $\mathrm{H}-\mathrm{A}-\mathrm{V}$ sequence. These features were diagnostic for slow-fast atrioventricular nodal reentrant tachycardia (AVNRT), excluding orthodromic reciprocating tachycardia (ORT) via an accessory pathway (AP) and atrial tachycardia. ${ }^{1,2}$ The $\mathrm{V}-\mathrm{A}-\mathrm{V}$ response was seen during RV overdrive pacing, which was also excluded atrial tachycardia. ${ }^{2}$ However, the atrial activation sequence gradually shifted, suggesting the presence of a left lateral AP (Figure 1). Similar to the atrial activation during RV overdrive pacing, the earliest atrial activation was seen in the distal CS region with RV pacing during sinus rhythm, which indicated the presence of a left lateral AP (Figure 2). The earliest atrial activation shifted to the HB region as the ventricular extrastimulus coupling interval decreased to $280 \mathrm{~ms}$ at a basic pacing CL of $600 \mathrm{~ms}$, which suggested retrograde conduction over the fast pathway.

In a setting with the presence of retrograde AP conduction, the most likely diagnosis would be ORT via an AP. In this case, however, a diagnosis of AVNRT with a bystander left lateral AP was reached based on the findings described above. We performed a left lateral AP ablation followed by a slow pathway ablation. After the AP ablation, we attempted to induce the clinical tachycardia by atrial extrastimulation with and without an isoproterenol infusion, but failed. After several junctional beats were seen during the empirical slow pathway ablation, any further tachycardia was rendered noninducible.

The teaching points in this case were as follows: (1) The presence of an AP does not necessarily mean that the AP is involved in the tachycardia circuit, and (2) a bystander AP may hinder the interpretation of the intracardiac electrograms during diagnostic pacing maneuvers.

When an AP is identified by RV pacing, we assume that the diagnosis may be AVRT. However, the presence of an AP does not necessarily mean that the AP is involved in the tachycardia circuit. Even if an AP is present, the tachycardia circuit should be confirmed by conventional diagnostic pacing maneuvers. Although several reports have described double tachycardias such as ORT via an AP and AVNRT with a bystander $\mathrm{AP},{ }^{3,4}$ there are no electrogram-tracings of RV overdrive pacing during AVNRT with a bystander AP. Diagnostic pacing may not have been applicable, possibly due to the repeated and spontaneous transition of the tachycardia form. Electrophysiologists should be aware of these observations through our experience. The tachycardia circuit and location of the bystander AP can quickly be determined by only one tracing.

There is some controversy over whether bystander APs should be eliminated. ${ }^{5}$ In this case, we eliminated the left lateral AP first for the correct diagnosis of the tachycardia circuit, which was hindered by the AP, although no evidence of the ORT was observed. The incidence of complications is related to the number of ablation targets, i.e., the more substrates, the more complications. ${ }^{5}$ Electrophysiologists should carefully determine the ablation strategy considering the risk and benefit of a bystander pathway ablation.

\section{Acknowledgments}

We thank Mr. John Martin for his help in preparing the manuscript.

\section{Reference}

1. Nagashima K, Watanabe I, Okumura Y, et al. Ventriculoatrial intervals $</=70 \mathrm{~ms}$ in orthodromic atrioventricular reciprocating tachycardia. Pacing Clin Electrophysiol . 2016;39:1108-1115.

2. Knight BP, Zivin A, Souza J, et al. A technique for the rapid diagnosis of atrial tachycardia in the electrophysiology laboratory.J Am Coll Cardiol . 1999;33:775-81.

3. Kuo JY, Tai CT, Chiang CE, et al. Mechanisms of transition between double paroxysmal supraventricular tachycardias. J Cardiovasc Electrophysiol . 2001;12:1339-45. 
4. Di Biase L, Bai R, Tritto M, Grimaldi M and Biasco MG. Ablation of atrioventricular nodal "slow pathway" for simultaneous treatment of coexisting atrioventricular and nodal reciprocating tachycardias. $J$ Interv Card Electrophysiol . 2007;19:143-7.

5. Calkins H, Yong P, Miller JM, et al. Catheter ablation of accessory pathways, atrioventricular nodal reentrant tachycardia, and the atrioventricular junction: final results of a prospective, multicenter clinical trial. The atakr multicenter investigators group.Circulation . 1999;99:262-70.

\section{Figure legends}

\section{Figure 1A-B}

Intracardiac electrograms with RV overdrive pacing during the tachycardia, and (B) the enlarged view of (A).

HRA p, high right atrium proximal; HRA d, high right atrium distal; HB p, His bundle proximal; HB d, His bundle distal; CS p, coronary sinus proximal; CS d, coronary sinus distal; RVA, right ventricular apex; A, atrial; H, His bundle; V, ventricle; TCL, tachycardia cycle length; PCL, pacing cycle length.

\section{Figure 2}

Intracardiac electrograms during RV extrastimulation. (A) The earliest atrial activation is seen in the distal CS with ventricular extrastimulus coupling intervals from 400 to $290 \mathrm{~ms}$ at a basic pacing CL of $600 \mathrm{~ms}$, suggesting the presence of a left lateral accessory pathway. (B) The earliest activation shifts to the HB region with a ventricular extrastimulus coupling interval of $280 \mathrm{~ms}$, suggesting the presence of retrograde fast pathway conduction.

Abbreviations are as in Figure 1.

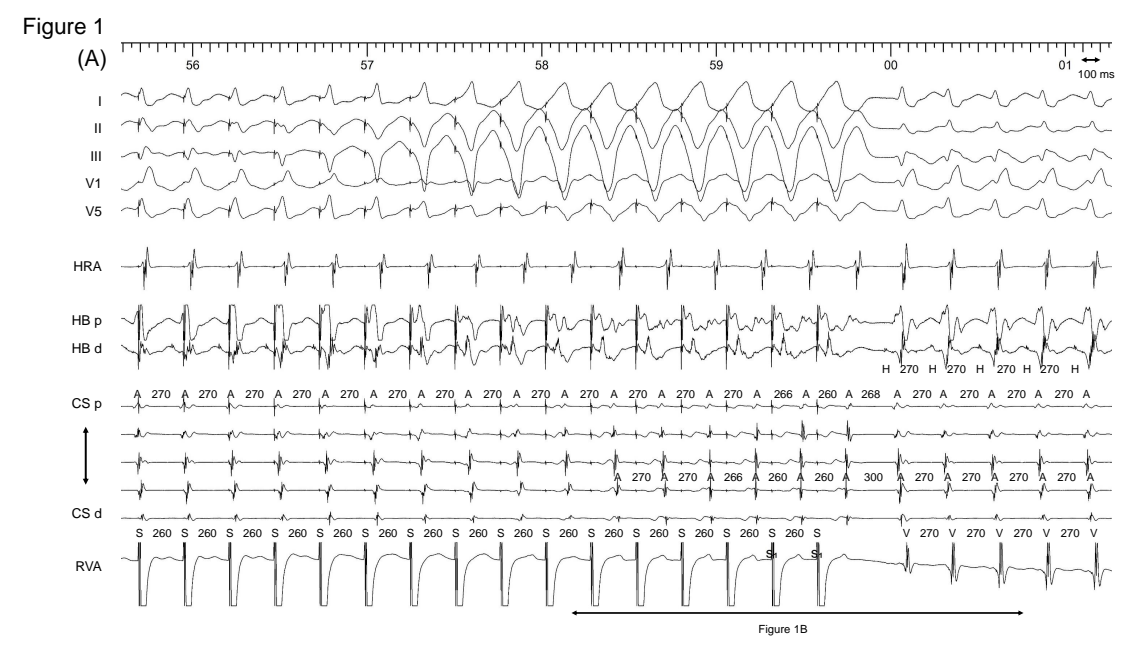




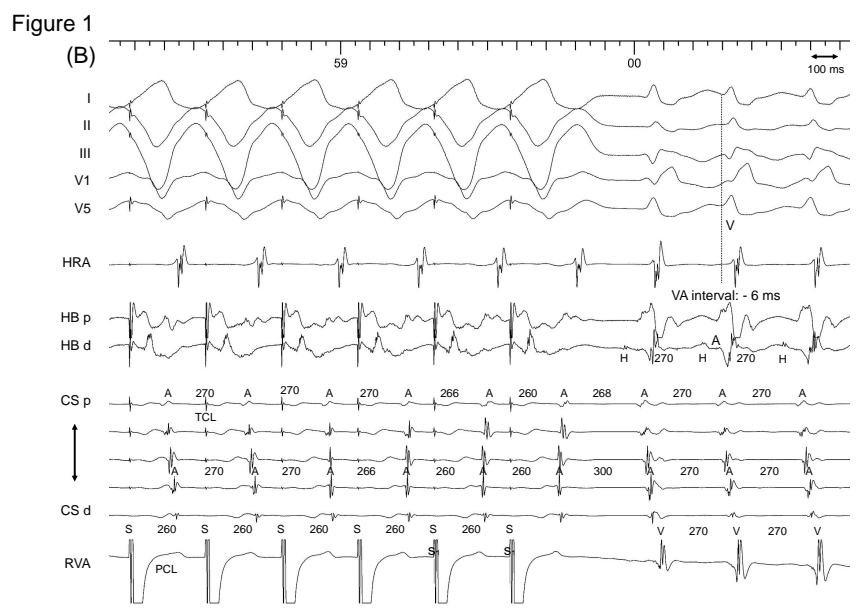

Figure 2

(A)

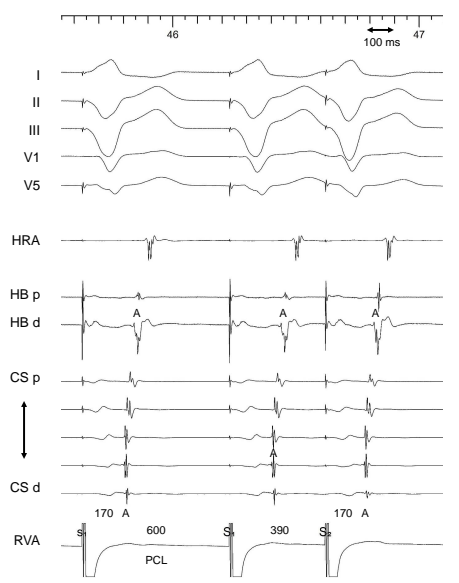

(B)

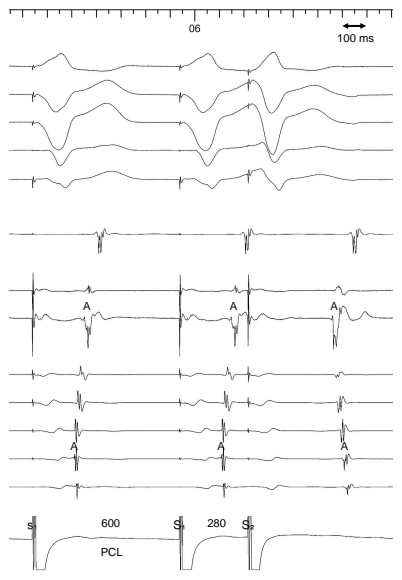

